\title{
Fractal analysis of colony margins as an aid for screening freshwater yeast cultures for bioclarification of turbid polluted water resources
}

\author{
Sheela Pal ${ }^{*}$ \\ Mycological Laboratory, Department of Botany, Goa University, Taleigao-403206 (Goa), \\ India \\ Nandkumar Kamat \\ Mycological Laboratory, Department of Botany, Goa University, Taleigao-403206 (Goa), \\ India \\ *Corresponding author. E-mail: nandkamat@gmail.com
}

\begin{abstract}
In Iron ore mining areas of Goa, water resources are polluted due to high turbidity and mineral colloids. For bioclarification of the turbidity, we need to identify some promising property of strains by which the strains of freshwater yeasts can be screened. This work presents a screening of freshwater yeast cultures, based on the complexity of colony margins. We performed screening of the wild aquatic yeasts isolated from different fresh water bodies of Goa on 2nd, 4th and 6th day of incubation respectively. Colony margins of sixteen different strains were studied for their fractality indexes and on comparison significant differences were observed among them. We report comparative analysis of five representative strains in this paper. Particularly strain Bchlm-1-2 showed high fractality index approximately 1410 on $6^{\text {th }}$ day of incubation. This work provides quantitative scoring system of the morphological behavior of large number of strains. Our approach has the potential to improve the accuracy and speed to quantify and compare large number of isolates on the basis of their colony margins.
\end{abstract}

Keywords: Biosedimentation, Colony margins, Flocculation, Fractal dimensions

\section{INTRODUCTION}

Fractal geometry has made important contributions in understanding the growth patterns of microbes. It permits measurement of the irregularity and complexity of mycelial surface structures correlated with growth, metabolic activity, enzyme production and pigmentation (Obert et al., 1990). Availability of nutrients can also be estimated with the help of fractal growth patterns. Puchkov (2016) has employed image analysis in the studies of both the macroscopic and the microscopic microbiological objects obtained by various imaging techniques. Low nutrient conditions produce low hyphal mass while high nutrient produce high hyphal mass (Gadd et al., 2001). Once the microbial mycelia start to grow, branches begin to emerge in different directions. Fractal behavior of microbial aggregation depends on the type of microbes and the method used to aggregate them (Logan, 1991). Mycelial morphology and its productivity such as citric acid fermentation and several antibiotics fermentations can be correlated with their fractal dimensions (Papagianni, 2004; 2006). Heterogeneities in colony and density, vacuoles within mycelia are not easy to calculate, therefore aver-

\section{Article Info}

DOI:10.31018/jans.v11i2.2028

Received: February 21, 2019

Revised: March 28, 2019

Accepted: April 5, 2019

\section{How to Cite}

Pal, S. and Kamat, N. (2019). Fractal analysis of colony margins as an aid for screening freshwater yeast cultures for bioclarification of turbid polluted water resources. Journal of Applied and Natural Science, 11(2): 250- 256 https://doi.org/10.31018/ jans.v11i2.2028 
pared with the help of fractal dimension of bacterial colony. Morphology of Achlya bisexualis in the response to heavy metal concentration, fractal dimension decreases as concentration increases (Lundy, 2001). Gonzalez-perez et al. (2016) mimicked the biofilm formation by polymerosomes and aggregation process and analysed it with fractal frame work and correlated it anti-bacterial property. Fractal dimensions can be good tool for studying the aggregates by providing quantitative measurement of aggregates morphology (Logan, 1991). However, Flocs cannot be studied due to their fragile nature. Particle aggregates generated in wastewater treatment process possess fractal features. (Li and Ganczarczyk, 1989). The fractal theory developed by Mandelbrot proposed new approach to define the geometry of those systems which have no definite geometry. The most important numerical parameter to calculate the fractal of any mass is fractal dimension. Fractal dimension is defined simply as the number of independent quantity needed to specify the position or arrangement of point on the object (Shirali, 2014). Many concepts have been proposed for the calculation of fractal dimension of any fractal mass. Low fractal dimension shows formation of aggregates due to cluster to cluster collision ( $\mathrm{Li}$ and Leung (2005), developed new method to determine fractal dimension of bio- flocs in activated sludge suspensions. Fractal dimensions have been used as tool to differentiate the flocs and granule particles formed in anaerobic digester used for water treatment (Bellouti et al., 1997). Saiedi et al. (2017) studied temporal variation in aggregates stabilization, carbon content and microbial respiration by the using theory of fractal geometry. Balaban (2018) investigated the evolution of Enterobacter cloacae aggregates evolution of spatial arrangement multifractal analysis of Enterobacter cloacae aggregates have been done to evaluate its radial growth on semi-solid media. Trichiurus haumela have been characterized by fractal dimension tool during frozen condition. During storage it has been observed that with decreasing the temperature its quality (hardness and springiness) decreased and its fractal dimension decreased as well (Luan et al. 2018). Soil structure depends on the soil particle association and mineral and organic matter due to which different types of aggregates form. Water retention by soil particles had been compared using fractal dimensions. Fractal dimensions of soil water retention curve was correlated with the soil bulk density and clay silt content (Mahallati et al., 2018). Fractal dimension concept has been used to describe the whey protein gel structure and its viscoelasticity. (Lestari, 2018). Malekani, (1996) compared fractal analysis technique for the determination of fractal dimensions of clay mineral. Fractal dimension of flocs fluctuate with changes in the mechanism of flocs formation. The fractal analysis theory can be used to understand the geometric characteristic of flocs (Li and Zhu, 2006). In biological flocs and the flocculation phenomenon, lowest fractal dimensions were observed due to bridging the aggregates, while higher fractal dimension observed aggregates formed by sweep substrate transfer. Hydraulic condition determines the fractal dimensions of flocs as well (Sun, 2013). In addition, initially it increases and then decreases after stirring. There are various methods to determine fractal dimensions all methods is based on power law relationship (Lee and Kramer, 2004). In previously reported work yeast colonies were characterized by its whole surface (Prado, et al., 2014). Growth of yeast colony with respect to colony height studied earlier (Ravindranath et al 1998). Morphological study of yeast on the basis of margins with image analysis has not been much done so far.

As a part of ongoing work to assess the feasibility of employing interesting strains of ascomycetous and basidiomycetous yeasts isolated from local freshwater ecosystem for bioclarification of turbid polluted water resources in Goa's Iron ore mining area, a need was felt for a reliable procedure to screen and select potentially useful strains. The present work was undertaken to check whether yeast colonies grown on solid media producing simple and complex margins could be subjected to fractal analysis and whether it could be an aid to select a superior strain. We tested the hypothesis that Fractal dimension of colony margins of yeasts could serve as an aid to screen and select strains capable of bioclarification of turbidity.

\section{MATERIALS AND METHODS}

One important tool used for the analysis was Fractality index. It is a four-digit number obtained by multiplying the score produced by the fractal analysis software like JFRAD by 1000 with last number being rounded up. The detailed description of the method is given below:

Water sample collection and isolation of yeast: Freshwater bodies of Goa from mining and nonmining areas were chosen for sample collection for isolation of yeast. The satellite images of water sampling sites are shown in Fig. 1. For the isolation of yeast cultures, samples of $500 \mathrm{ml}$. water were collected in pre-sterilized plastic bottles. The Isolation of yeast cultures was carried out by spread plate method on MEA medium after passing the water sample through membrane filter. Filter papers were soaked in $3 \mathrm{ml}$ of sterile water. Faropanem (MEA plates with $0.1 \%$ Faropenem) used as antibiotic to avoid the bacterial growth. Plates were incubated at $24-25{ }^{\circ} \mathrm{C}$, observed under microscope. Dissimilar colonies were picked and streaked on purification plate and re-streaked on MEA slants to maintain the culture. Colony of 
isolates classified on the basis of simple colony margin and complex colony margins. Colonies with complex colony margins were studied for their fractality index. Yeast strain were streaked on solid media in nested colony square pattern in 9 c.m. petriplates which facilitated observation of colonies under microscope. Nested colony patterns avail to observe the interaction of colony margin on the same media inoculated at the same time. Adaptation against poor nutrient condition can also be observed.

Selection of strains for assay: All isolates were observed systematically and classified on the basis of simple colony margin and complex colony margin. Isolates were classified on the basis of regular and irregular growth of margins on solid medium. Preliminary isolates with complex colony margins were studied for analysis of their fractality index. Among all isolates with complex margin, the top five isolates with the highest fractality indices were studied and compared for their biofloculation capability of turbid water. Compactness of floc and rapid sedimentation of clay colloid were compared of the isolates.

Digital analysis of colony margins: Pure yeasts cultures isolate which were showing compact floc formation and having complex fractal margins were grown on thinner layer of $2 \% \mathrm{MEA}$, in a nested square pattern, Please refer to Fig.1, to produce defined colonies and the margins were photographed at regular time intervals up to 7 days. Representative images were imported and processed to compute fractal dimension employing CMEIASJFrad version 1.0 software (available at http://cme.msu.edu/cmeias/ (Ji et al., 2015)) which uses 11 different mathematical methods. The output data of yeast colony fractal dimensions were saved as a * $\mathrm{Csv}$ files and analyzed statistically using the SYSTAT 13. Fractality indices of the margins were calculated using fractal dimension of colony margins and compared.

\section{RESULTS}

Selected strains have their specific colony characteristics (Table 1). Yeast isolates with complex fractal margins are given in Fig. 3 for five culture designations. Each isolate has its specific colony margin and the emergence of the margin rate varies as a function of time. These colonies grew according to the predetermined pattern as outlined in Fig. 2.

Table 1. Characteristics of selected yeast strains.

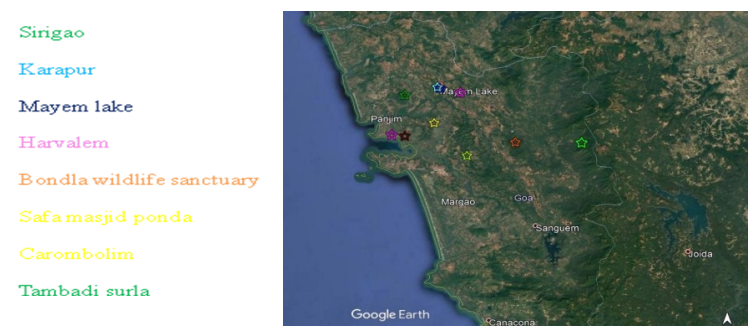

Fig.1. Satellite images of water sampling sites (in right) are represented by star of the same color as their respective places (in left).

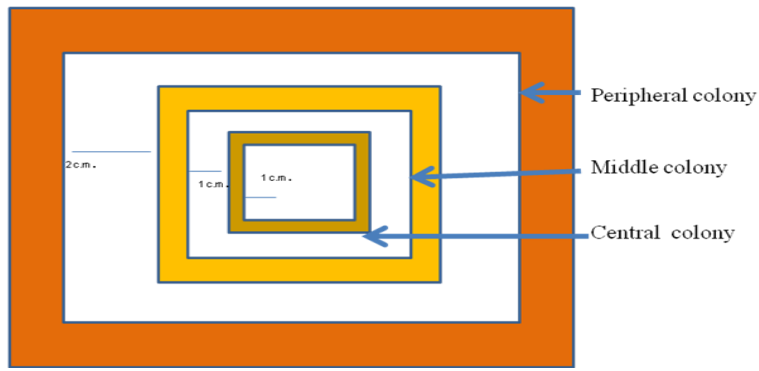

Fig.2. Predetermined pattern to obtain colonies amenable to digital analysis.

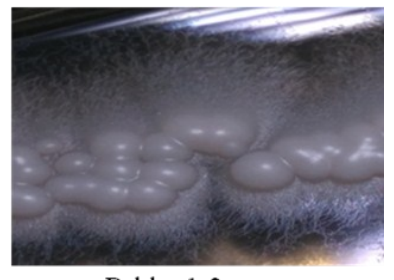

Bchlm-1-2

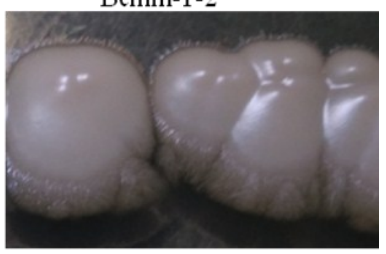

Pnd-2

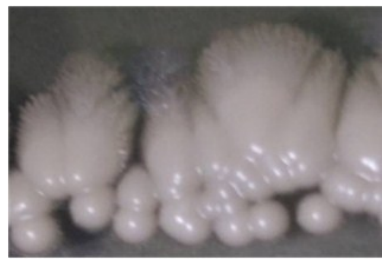

Gh1-1

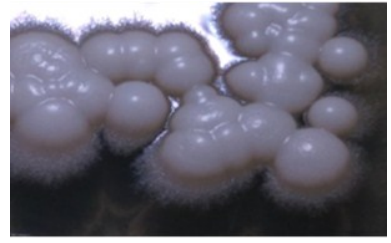

Pndsm-2-2

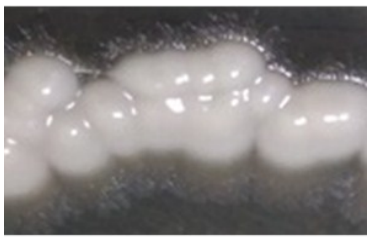

Tmrs-2-2-4

Fig.3. Yeast isolates with complex fractal margin's photographs each showing specific colony margin.

The amplified picture of yeast strains taken on $6^{\text {th }}$ day is shown in Fig. 4 to show the fractal margins of the five strain designations. Micromorphology of

\begin{tabular}{|c|c|c|c|c|c|c|}
\hline $\begin{array}{l}\text { Culture } \\
\text { Designation }\end{array}$ & Colony & Texture & Surface & Elevation & Color & Margin \\
\hline Bchlm-1-2 & Circular & Waxy & Smooth & Flat & white & Complex \\
\hline Gh1-1 & circular & Shiny & Smooth & Flat & white & Complex \\
\hline Pnd-2 & Granular & Shiny & Smooth & Flat & Pinkish & Complex \\
\hline Pndsm-2-2 & Circular & Shiny & Smooth & Flat & white & Complex \\
\hline Tmrs-2-2-4 & Circular & Waxy & Smooth & Flat & Beige & Complex \\
\hline
\end{tabular}


Pal, S. and Kamat, N. / J. Appl. \& Nat. Sci. 11(2): 250- 256 (2019)

Table 2. Fractality Index of colony margins at different time intervals.

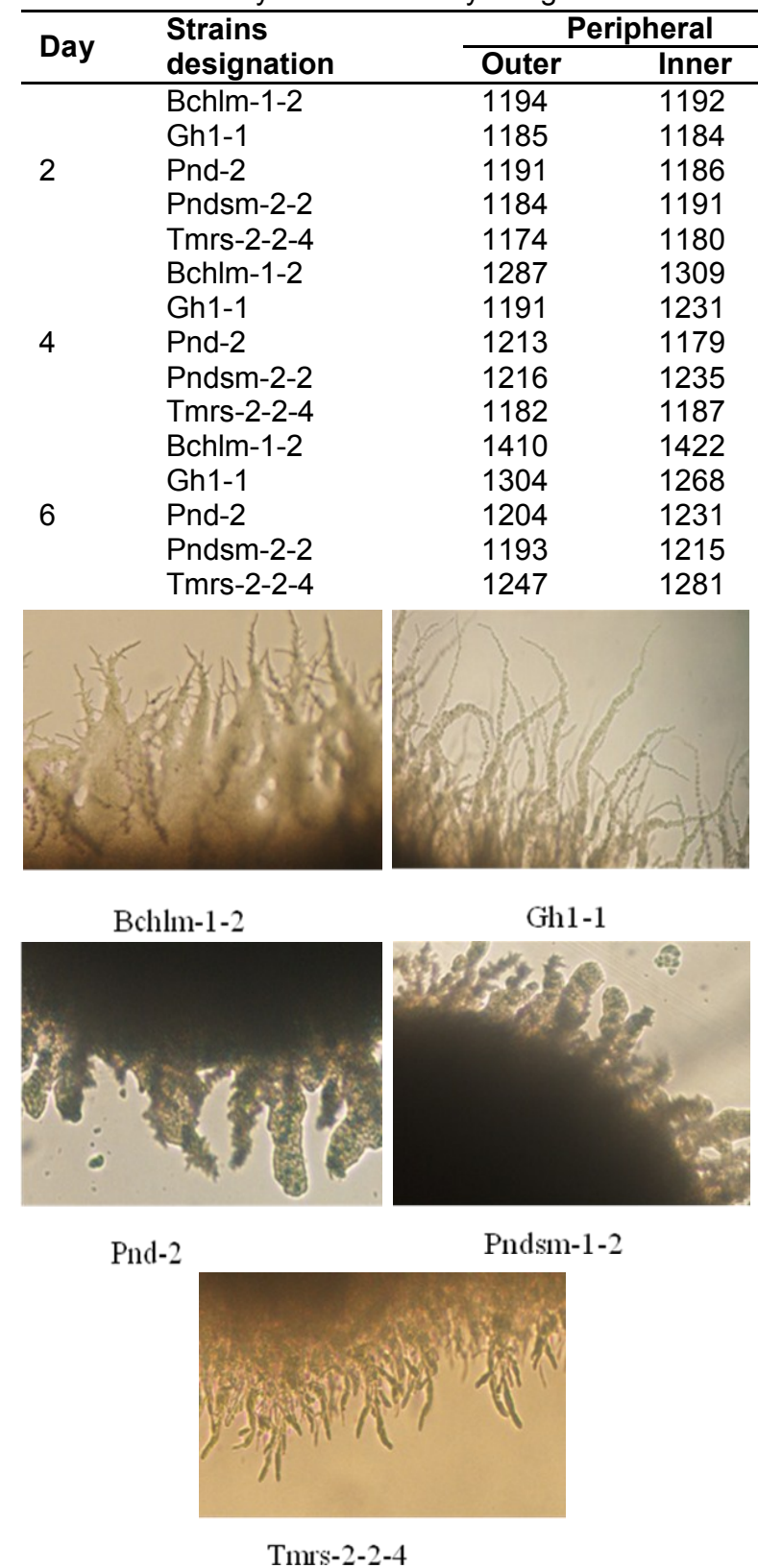

Fig.4. Fractal margins of yeast strains under $10 x$ objective lens taken at $6^{\text {th }}$ day of incubation.

colonies margins showed variation in the colony margin among the isolates. It was observed that Strain designated as Bchlm-1-2 showed high growth rate of the complexity of colony margins and complexity on its edges within $24 \mathrm{hr}$ of inoculation. Moreover, this strain showed more complex growth of margins with respect to time.

Strain designated as Gh1-1 showed less complexity on the surface of colony margin on the second day. However, its colony margin showed very complex structure after 3 days.

Strain designated as Pndsm-1-2 showed smooth

\begin{tabular}{cc} 
& Middle \\
\hline Outer & Inner \\
\hline 1194 & 1225 \\
1186 & 1181 \\
1207 & 1219 \\
1183 & 1190 \\
1178 & 1190 \\
1302 & 1309 \\
1227 & 1231 \\
1226 & 1289 \\
1230 & 1249 \\
1180 & 1258 \\
1357 & 1408 \\
1288 & 1230 \\
1242 & 1278 \\
1305 & 1216 \\
1269 & 1366 \\
& \\
& \\
& \\
& \\
&
\end{tabular}

\begin{tabular}{lc} 
& Centre \\
\hline Outer & Inner \\
\hline 1224 & 1281 \\
1185 & 1212 \\
1184 & 1238 \\
1177 & 1186
\end{tabular}

Bchlm-1-2

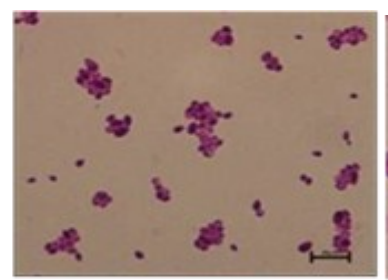

$1177 \quad 1186$

$1054 \quad 1179$

$1300 \quad 1311$

$1262 \quad 1205$

$1231 \quad 1226$

$1235 \quad 1311$

$1207 \quad 1183$

$1425 \quad 1397$

$1280 \quad 1326$

$1221 \quad 1256$

$1269 \quad 1353$

$1331 \quad 1277$

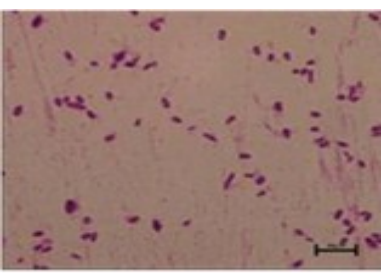

Bchlm-1-2

Gh 1-1

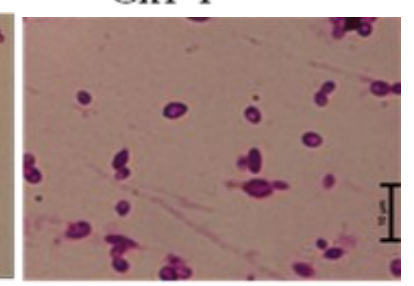

Pnd-2

Pndsm-2-2

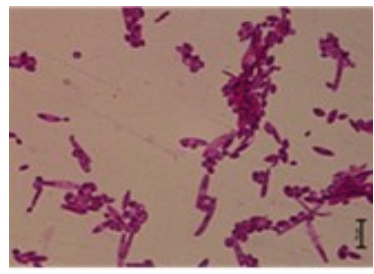

Tmrs-2-4

Fig.5. Micromorphology of yeast strains, Bchlm-12 strain cells were stained lightly and showed single and budding cells with bi and multipolar budding. Gh1-1 strains cells were smaller than other isolates and showed single and budding cells. Pnd -2 showed cluster of single cells and darkly stained. Pndsm-2-2 showed single and budding cells large in size and darkly stained. Tmrs-2-4 showed single cells and pseudo-mycelium.

edges at the beginning but after three days it exhibited complex growth of colony margins with respect to time.

Strain designated as Pnd-2 showed slow growth rate of its complexity of margins and after three days with irregular and complex colonies with in- 


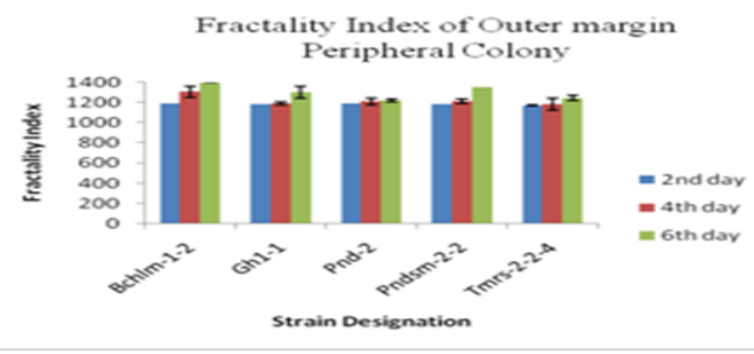

(a)

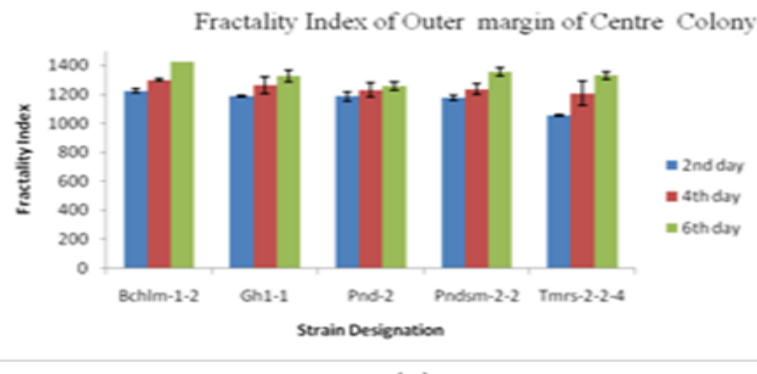

(c)

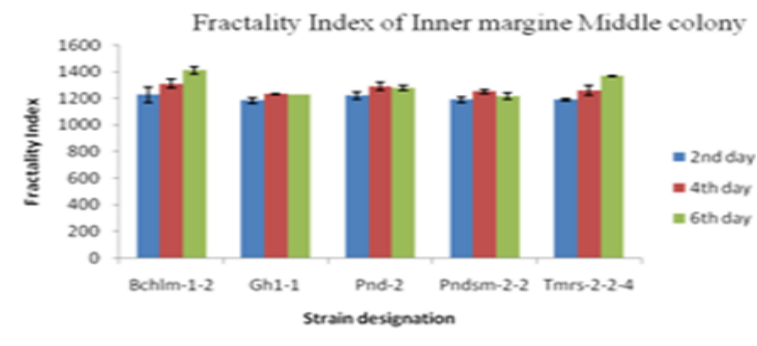

(e)

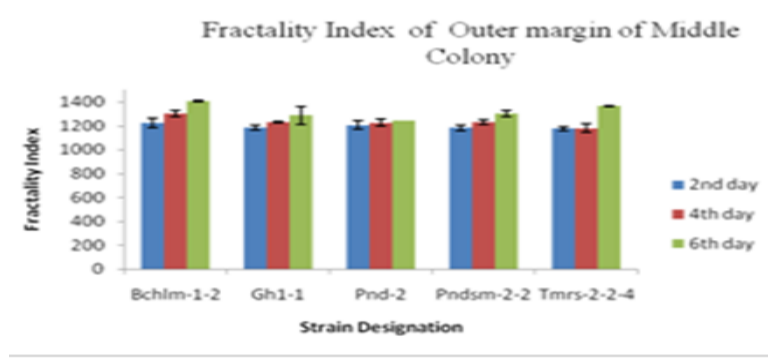

(b)

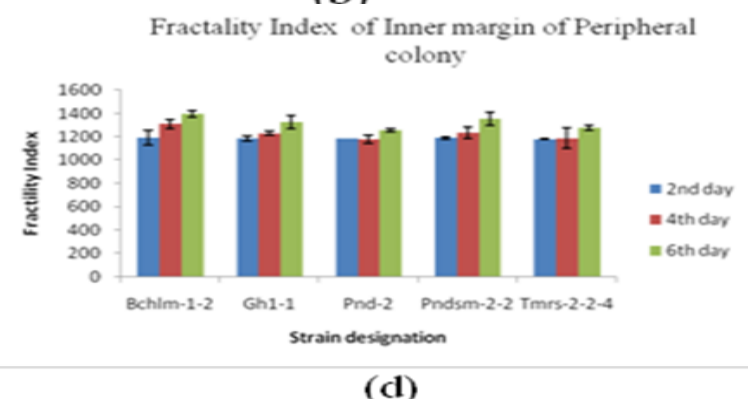

Fractility Index of Inner margin of Centre Colony

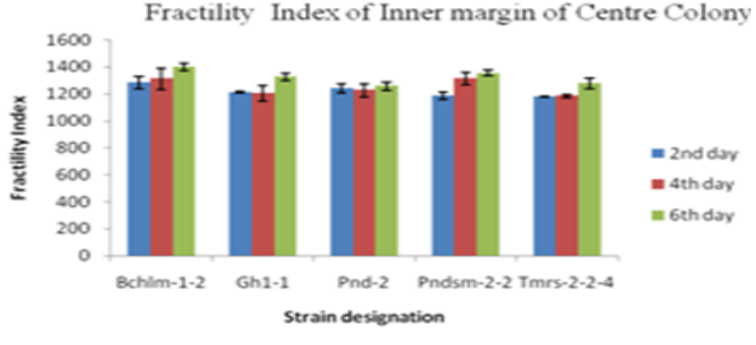

(f)

Fig.6. Fractality Index of colony margins as funtion of growth (a) Outer margin of peripheral colony (b) Outer margin of middle colony (c) Outer margin of central colony (d) Inner margin of peripheral colony (e) Inner margin of peripheral colony (f) Inner margin of central colony.

creasing complexity with respect to time.

The isolates Tmrs-2-2-4 showed complex colony edges within two days.

Micromorphology of isolated strains (Fig.5) showed variation in their cellular size and shape as they are circular, ovale in shape and some are dimorphic in nature, single cell, and pseudomycielium as well.

Fractality index of strains margins increased with respect to time. Internal margins of central most colony showed high fractality index except Bchlm1 -2 in which fractality index was highest of outer margins of peripheral colony. Central colony margins of Bchlm-1-2, Gh1-1, Pnd-2 grew faster initially. On $4^{\text {th }}$ and $6^{\text {th }}$ day, Central colony margins growth rate was almost same as peripheral and central colony. While Pndsm-2-2-4 and Tmrs-2-2 peripheral colony margins were more complex with high fractality index on the $2^{\text {nd }}$ day. Fractality index was highest on $6^{\text {th }}$ day compare to second day of inoculation of colonies of all isolates.

All strains showed different fractality index as they had different kind of complex fractal margins and varied in growth pattern. Most of the strain margin were showed high fractality index which were interacted parallel to each other. Among all strains, Bchlm-1-2 showed consistent in highest fractality index (Table 2). Among all five strains, Bchlm-1-2 showed compact and large floc formation and rapid sedimentation of clay colloids in turbid water.

\section{DISCUSSION}

The captured photograph of colony margins of freshwater yeast isolates is presented in Fig. 3 and their fractality indices are compared in terms of the growth functions and their respective positioning (Fig. 6). The results are reliably consistent with our preliminary study conducted in the laboratory by using the all isolates.

Generally, yeasts tend to grow in set patterns and even after streaking they do not spread all over the plates. In present study, colonies were grown in nested patterns as in Fig.2 and observed them under the microscope (objective lens 10X) by using a $9 \mathrm{~cm}$. petriplate. Predetermined nested pattern of grown colonies on solid media was easy to observe, capture photograph and generate random data set. Moreover, square pattern has pro- 
vided linear growth of replicated colonies.

Growth of colonies margins were observed and characterized with temporal and spatial variations. Since colonies were inoculated on the same day in the same media, growth and morphological behavior could easily compared for each colony.

We observe that three nested colonies were growing in the same pattern increasing in complexity in their margins with respect to time, but their fractality indices varied with respect to position. The underlying cause could be heterogenesity of colonies or internal switch in genes to survive in nutrient deficient medium. Increase in the fractality index with increase in the margin complexity was also observed. Further study of colony margins and their interactions can be done to find out the cause of variation in growth patterns because these characteristics may be ruled by chemo-taxis interaction or gene expressions.

Fractality index analysis of isolates colony margin is proved to be useful aid to characterize freshwater yeast strains and utilize the fractal dimensions for establishing a clear positive or negative correlation with their other biotechnical potential. Very little information is available on the fractal analysis of oligotrophic freshwater yeast and its correlation with their application potential. Wherever previously Ruusuvuori et al. (2014) performed quantitative analysis of yeast colony morphology using image-based techniques. This method encourages the computational classification tools for the analysis of colony shapes. They observed spatiotemporal dynamic study of yeast colonies, extracted from this tool had different value for smooth and fluffy colonies. Gontar (2018) and his coworker analysed colony morphology of Saccharomycese cerevisiae using two dimensional top down binary images, cluster shape primitive tool (Csps) and provided promising approach for analyzing colony shape in high throughput assay. Further, they Suggested this tool provides a medium of identifying and quantifying the specific shape. Present study provides comparative analysis of different isolate's colony margins and their application in biosedimentaion of clay colloids. This technique could be further used for the analysis of other microbes like bacteria and fungi.

Fractal analysis of colony margins of these freshwater yeast isolated from Goa's different fresh water reservoirs have been studied for the first time. This study showed that the image analysis method to study fractality index of colony margins could be great tool to rapid screening of yeast isolates for biosedimentation of clay colloids of turbid waters. This image analysis based technique holds excellent potential for rapid screening of a large number of yeast strains required in different biotechnological applications e.g. growth, metabolite production.

\section{Conclusion}

In this study we employed an image based technique by using CMEAS JFRAD to quantify strains margins and we observed that isolates showed specific growth patterns of their respective colony margins on same source of growth media. Their respective fractality indexes and colony margin structures were observed to be significantly different. We observed that Bchlm-1-2 showed the most complex colony margin and the highest fractality index of all considered strains. This study helps to understand how colony margin structure of each isolate grows with respect to time. In this work we studied only wild freshwater yeast isolates. However, our approach of using CMEAS JFRAD can be extended for the study of other microorganisms bacteria, fungi as well.

\section{REFERENCES}

1. Balaban, V., Lim, S., Gupta, G., Boedicker, J., and Bogdan, P. (2018). Quantifying emergence and selforganisation of Enterobacter cloacae microbial communities. Scientific Reports, (January), 1-9.

2. Barry, D., and Mcgee, S. (2009). Relating Fractal Dimension to Branching Behaviour in Filamentous Microorganisms, ISAST Transactions on Electronics and Signal Processing, 4 (1): 71-76.

3. Bellouti, M. (1997) Flocs vs granules : differentiation by fractal dimension. Water Research, 31:5 12271231

4. Boddy L, Wells J. M., Culshaw C, Donnelly D.P. (1999). Fractal analysis in studies of mycelium in soil. Geoderma, 88:301-328.

5. Gadd, G. M., Ramsay, L., Crawford, J. W. and Ritz, K. (2001). Nutritional influence on fungal colony growth and biomass distribution in response to toxic metals. FEMS Microbiology Letters, 204(2), 311-316.

6. Gonzalez-perez, A., Feld, K., and Ruso, J. M. (2016). Polymersomes mimic biofilms fractal growth. Journal of Polymer Research, 1-6.

7. Gontar A, Bottema M.J., Binder, B.J., Tronnolone, H. (2018). Characterizing the shape patterns of dimorphic yeast pseudohyphae. $R$. Soc. Open Sci., 5: 180820

8. Ji, Z., Card, K.J. and Dazzo, F.B. (2015) CMEIAS J Frad: A digital computing tool to discriminate the Fractal geometry of landscape architectures and spatial patterns of individual cells in microbial biofilms, Microb Ecol., 69:710-720

9. Lestari, V. D., Mardawati, E., and Nurhadi, B. (2018). Fractal Dimension Analysis of Texture Formation of Whey Protein-Based Foods, International Journal of Food Science Volume 2018, Article ID 7673259,1- 17

10.Lee, C., and Kramer, T. A. (2004). Prediction of three -dimensional fractal dimensions using the twodimensional properties of fractal aggregates, Advances in colloid and Interface Science, 112, 49-57.

11.Li, D. H., and Ganczarczyk, J. (1989). Fractal geometry of particle aggregates generated in water and wastewater treatment processes. Environmental Science and Technology, 23(11), 1385-1389.

12. Li, T. Z. Zhu, D.S. (2006) Wang, C.H. Yao, H.X. Tang, Characterization of floc size, strength and structure under various coagulation mechanisms, 
Powder Technol., 168 104-110.

13.Li, X. Y., and Leung, R. P. (2005). Determination of the fractal dimension of microbial flocs from the change in their size distribution after breakage. Environmental Science and Technology, 39(8), 27312735.

14.Logan, B. E. (1991.). Fractal Coagulation Kinetics. Department of Civil and Environmental Engineering The Pennsylvania State University.

15.Luan, L., Sun, Y., Chen, S., Wu, C., and Hu, Y. (2018). A study of fractal dimension as a quality indicator of hairtail ( Trichiurus haumela ) samples during frozen storage. Scientific Reports, (December 2017), $1-8$.

16.Lundy, S. D., Payne, R. J., Giles, K. R., and Garrill, A. (2001). Heavy metals have different effects on mycelial morphology of Achlya bisexualis as determined by fractal geometry, FEMS Microbiology Letters, 201 201, 259-263.

17.Mahallati, S. Z., Pazira, E., Abbasi, F., and Babazadeh, H. (2018). Estimation of soil water retention curve using fractal dimension. Journal of Applied Sciences and Environmental Management, 22(2), 173-178.

18.Malekani, K., Rice, J. A., and Lin, J. (1996). Comparison Of Techniques For Determining The $F$ ractal Dimensions of clay mineral, Journal of Clays and Clay Minerals, 44(5), 677-685.

19.Obert, M., Pfeifer, P., Sernetz, M., and Giessen, J. (1990). Microbial Growth Patterns Described by Fractal Geometry, Journal of Bacteriology, 172(3), $1180-1185$.

20.Obert. M. (1993). Microbial growth patterns: fractal and kinetic characteristics of patterns generated by a computer model to simulate fungal growth. Journal of Fractal Biology, 254-374.

21.Papagianni, M. (2006). Quantification of the fractal nature of mycelial aggregation in Aspergillus niger submerged cultures, Microbial Cell Factories, 13:1-13

22.Papagianni M: (2004). Fungal morphology and me- tabolite production in submerged mycelial processes. Biotechno.l Adv. 22:189-259.

23.Prado, E. G. De, Rivas, E., Silóniz, M. De, Diezma, B., and Barreiro, P. (2014). Quantitative analysis of morphological changes in yeast colonies growing on solid medium: the eccentricity and Fourier indices, Yeast 2014; 31: 431-440.

24.Puchkov, E. (2016). Image Analysis in Microbiology: A review. Journal of Computer and Communications, $4: 8-32$.

25.Ravindranath S., Kamath, Bungay H,..(1998). Yeast colonies on solid media, Journal of General Microbiology (1988), 134, 3061-3069.

26.Ruusuvuori, P., Nykter, M., Yli-harja, O., Shmulevich, I., and Dudley, A. M. (2014). Quantitative analysis of colony morphology in yeast. Biotechniques. 2014 January ; 56(1): 18-27 56(1).

27.Saiedi, N.N., A. A. Besalatpour, H. Shirani, P. Abbaszadeh Dehaji, I. Esfandiarpour, M. Faramarzi (2017). Agregation and fractal dimension of aggregates formed in sand dunes stabilized by Pistachio PAM and PistachioPVAc, European Journal of Soil Science, 783-791.

28. Shirali, S. A. (2014). Fractal dimension and the cantor set, Resonance 1000-1004.

29.Sun, J., Qin, L., Li, G., and Kang, Y. (2013). Effect of hydraulic conditions on flocculation performances and floc characteristics in Chinese herbal extracts by chitosan and chitosan hydrochloride. Chemical Engineering Journal, 225: 641-649.

30.Tomankova, K. (2006). Use of image analysis to study growth and division of yeast cells, ImagingSci.Technol.50: 583-589

31.Vesela, M., Zemskal, O., Vesely, M., Nezadal, M. (2001). The fractal analysis of image structures for bilogic applications, Digital Imagine in Biology and Medicine, 53-54.

32.Woriax, H. E. (2009). A study on fractal morphogenesis in bacteria as a response to environmental stress Hannah E . Woriax Honors College Thesis UNC Pembroke, 1-18. 